\title{
La liaison variable chez les locuteurs français septentrionaux et méridionaux
}

\author{
Julia Steele Josephs ${ }^{1,{ }^{*}}$ et Diana L. Ranson ${ }^{2}$ \\ ${ }^{1}$ Department of Linguistics, University of Georgia, Athens, GA, 30602, États-Unis \\ ${ }^{2}$ Department of Romance Languages, University of Georgia, Athens, GA 30602, États-Unis
}

\begin{abstract}
Résumé. Le but de la présente étude est de décrire le statut de la liaison en français moderne en fonction des caractéristiques des locuteurs, surtout leur lieu d'origine, et des facteurs linguistiques. Une analyse de 2.498 sites de liaison produits par 40 locuteurs du projet Phonologie du français contemporain et du Corpus Montpellier-Rognes montre que les locuteurs âgés du sud affichent un taux de liaison plus élevé que celui des jeunes locuteurs du sud et de tous les locuteurs du nord. Le sexe des locuteurs, cependant, n'a pas d'effet significatif sur les résultats et la différence selon la région est significative seulement chez les locuteurs âgés. Les résultats selon la classe grammaticale du mot liaisonnant montrent que certaines liaisons, comme après très and dans, se font presque toujours, tandis que d'autres restent variables, comme après quand et les formes du verbe être. D'autres liaisons ne se font que rarement, comme après les noms pluriels, pas et jamais, et d'autres formes verbales. En ce qui concerne la consonne de liaison, /t/ se trouve plus souvent dans les liaisons qui restent variables et $/ \mathrm{z} /$ dans les liaisons fréquentes et rares.
\end{abstract}

\begin{abstract}
Variable liaison by Northern and Southern French speakers The goal of the present study is to describe the status of liaison in Modern Spoken French according to speakers' characteristics, especially their place of origin, and linguistic factors. An analysis of 2498 potential liaisons in the Phonologie du français contemporain project and the Montpellier-Rognes Corpus shows that older Southern speakers maintain a higher rate of liaison than younger Southern speakers and Northern speakers. The speakers' sex, however, does not have a significant effect on the results and the difference by region is significant only for the older speakers. The results according to the grammatical category of the liaison word show that certain liaisons, such as those after très 'very' and dans 'in', are almost always realized, whereas other liaisons remain variable, like after quand 'when' and the forms of the verb être 'to be'. Other liaisons are made only rarely, including those after plural nouns, pas 'not' and jamais 'never', and verbs other than être. The liaison consonant is more often $/ \mathrm{t} /$ in variable liaisons and more often $/ \mathrm{z} /$ in frequent and rare liaisons.
\end{abstract}

*Corresponding author: JuliaSteeleJosephs@uga.edu 


\section{La liaison dans une perspective historique}

La liaison, la prononciation d'une consonne latente devant une voyelle, comme le /t/ de est dans on est allé, représente du point de vue historique un des rares exemples de la survie des consonnes finales. En ancien français toutes les consonnes finales écrites étaient prononcées bien qu'elles soient souvent muettes en français moderne. Par exemple, le mot est se prononçait /عst/ non seulement devant voyelle (Li empereres est en un grant vergier, v. 103), mais aussi devant consonne (Li empereres est par matin levét, v. 163) (exemples tirés de La Chanson de Roland, Segre et al. 2003). Au cours des siècles suivants, la consonne en fin de mot s'est perdue progressivement, d'abord devant un mot commençant par une consonne, ensuite devant une pause, et finalement devant un mot à initiale vocalique dans certains contextes syntaxiques. Au $16^{\mathrm{e}}$ siècle les grammairiens recommandaient encore la prononciation invariable d'un $s$ en fin de mot devant une voyelle (Du Guez [1532], cité par Pope 1934 :220 ; Sylvius (Jacques Dubois) [1531], cité par Walter 1988:91; Saint-Liens [1580], cité par Thurot 1881 :7). Cependant, vers la fin du $17^{\mathrm{e}}$ siècle, les conseils des grammairiens indiquaient que cette prononciation était devenue variable. Hindret [1687] recommandait la prononciation dé jan inconu plutôt que dèjanz inconu pour des gens inconnus et Antonini [1753] préférait les prononciations "ils von à Rome, des spectacl' incroyables, et des plaisir infinis » et pour éviter "l'affectation » dans la conversation (cités par Thurot 1881 :9-10). Le phénomène moderne de la liaison, qui semble synchroniquement comme l'ajout d'une consonne, est donc historiquement la conservation des consonnes finales devant certains mots commençant par une voyelle. Cette conservation sélective des consonnes explique pourquoi de nos jours on peut conserver les consonnes finales $/ \mathrm{z} /, / \mathrm{t} /, / \mathrm{n} /$, et plus rarement $/ \mathrm{p} / \mathrm{et} / \mathrm{b} /$, en liaison et pourquoi les locuteurs réalisent toujours certaines liaisons dites invariables ou obligatoires, comme entre déterminant et nom (les amis), entre pronom et verbe (nous avons), ou entre verbe et pronom (allez-y), mais ne réalisent que quelquefois les liaisons dites variables ou facultatives, comme entre le verbe être et son prédicat (c'est une langue) ou entre avoir auxiliaire et son participe passé (ils ont appris). L'objectif de la présente étude est de décrire la liaison en français parlé pour fournir une évaluation actuelle de la perte de la consonne en fin de mot.

Pour analyser l'état actuel de la liaison, nous proposons de comparer la réalisation des liaisons variables dans des conversations quotidiennes avec 40 locuteurs français regroupés en fonction de leur sexe (femmes ou hommes), âge (32 ans et moins ou 40 ans et plus), et lieu d'origine (du nord ou du sud de la France). La grande majorité des études antérieures qui ont considéré la corrélation entre l'âge des locuteurs et le taux de liaison ont trouvé que les jeunes faisaient moins souvent la liaison que les locuteurs plus âgés. Ce résultat est valable pour les liaisons variables prononcées par des locuteurs de Tours enregistrés en 1976 (Ashby 1981 :54) et pour les liaisons après est, sont et suis prononcées par d'autres Tourangeaux en 1981 dans des interviews et dans les expressions, depuis un mois, depuis un an, et pendant un moment, dans une lecture et dans une liste de mots (Booij et De Jong 1987 :1017). La même distinction en fonction d'âge se trouve aussi chez des locuteurs méridionaux enregistrés en 2005 et 2006 (Ranson 2008 :1678) et chez des locuteurs enregistrés dans le cadre du projet Phonologie du français contemporain (PFC) dans différentes villes de France (Pagliano et Laks $2005: 4$, Mallet $2008: 194$, Durand et al. $2011: 127$, Eychenne 2011 :92) et dans la capitale ivoirienne, Abidjan (Walhout 2017 :35). Cependant, parmi les trois tranches d'âge de locuteurs à Chlef en Algérie (Walhout $2017: 35$ ) et de différents endroits en Suisse (Bergen 2005 :3), la tranche la plus âgée affiche le plus haut taux de liaison, comme dans la plupart des études, mais le groupe moyen a un taux de liaison plus bas que celui des plus jeunes. Les résultats selon l'âge sont moins 
nets chez un groupe de Parisiens qui affichent les taux suivants pour les liaisons invariables et variables confondues selon la tranche d'âge : 20-29 ans (63\%), 30-39 ans $(58,5 \%), 40-49$ ans $(63,9 \%), 50-59(69,3 \%)$, et 60-69 (67,5\%) (Malécot 1975 :169). Seulement deux groupes de locuteurs présentent des résultats contraires. Parmi les trois tranches d'âge des locuteurs à Bangui en République centrafricaine, ce sont les jeunes qui ont le plus haut taux de liaison (Walhout 2017 :35). Le même résultat se trouve chez des Français de différentes villes enregistrées entre 1998 et 2003 à Aix-en-Provence dans le cadre du corpus C-Oral-Rom (Meinschaefer et al. $2015: 388-89$ ). Les locuteurs de moins de 40 ans ont un taux de liaison de $32 \%$ contre 24\% pour leurs aînés.

Malgré la presque unanimité des résultats sur l'effet de l'âge sur la réalisation de la liaison, il n'y a pas d'unanimité sur la cause de ces résultats. Les deux explications possibles, notées par Booij et De Jong (1987:1016), Pagliano et Laks (2005:4), et Durand et Lyche $(2016: 375)$ et résumées par Nardy (2008:116-18) sont celle d'un changement en cours en temps apparent vers l'éventuelle perte de la consonne de liaison ou celle d'une stratification en fonction de l'âge ('age-grading'), selon lequel les jeunes locuteurs feraient plus de liaisons quand ils seraient plus âgés à cause de leur plus grande intégration dans la vie professionnelle. Durand et Lyche (2016:375) observent avec raison que ces deux explications ne sont pas mutuellement exclusives. Certains jeunes locuteurs pourraient réaliser plus souvent certaines liaisons en vieillissant en même temps que la majorité des locuteurs dans une communauté réalisent moins souvent d'autres liaisons. Tout compte fait, il serait prudent de ne pas baser la conclusion d'un changement en cours sur le simple fait d'un plus bas taux de liaison chez les jeunes locuteurs.

En analysant la liaison chez des locuteurs du nord et du sud de la France, il sera possible de voir le statut de la liaison sur le territoire français. Il est bien connu que certains locuteurs méridionaux font preuve d'un conservatisme linguistique par rapport à ceux du nord en prononçant parfois un schwa en fin de mot (bonne fête ) ou au milieu du mot (finement) et un appendice consonantique après une voyelle nasale, comme dans pain prononcé comme [pẽy]. Historiquement le schwa est une des dernières traces des voyelles finales ou médianes autrement perdues, comme dans les changements du latin BONA FESTA > bonne fête et de FINA MENTE > finement. Les voyelles nasales en français moderne ont évolué d'une séquence de voyelle plus consonne nasale dans laquelle la consonne nasale a transféré sa nasalité à la voyelle précédente avant de se perdre, comme dans PANEM > pain. Vu ces différences entre le nord et le sud, nous prédisons que les locuteurs septentrionaux auront un plus bas taux de liaison que leurs homologues méridionaux. Cette hypothèse suit les résultats de Durand et Lyche (2008:47-48) que les locuteurs méridionaux affichaient un plus haut taux de liaison que les locuteurs du nord de la France dans certaines formes du verbe etre. Nous proposons en plus que les jeunes des deux régions auront un taux de liaison plus bas que leurs homologues âgés.

Il sera important aussi de prendre en ligne de compte la classe grammaticale des mots entre lesquels la liaison est faite et la consonne de liaison. Cela nous permettra de déterminer si certains sites de liaison et certaines consonnes favorisent encore la réalisation de la liaison ou si, au contraire, la liaison de certaines consonnes dans certains contextes ne se fait plus. Les études antérieures ont montré que certains contextes favorisent la liaison variable, comme après une préposition et après le verbe être, tandis que d'autres contextes la défavorisent, comme après d'autres verbes et les noms pluriels (Malécot $1975: 164$, Ashby 1981 :50, Ranson $2008: 1679$ ). En ce qui concerne la consonne de liaison, les études sont d'accord que la liaison variable ne se fait plus avec la consonne $/ \mathrm{k} /$ et ne se fait que très rarement avec $/ \mathrm{s} / \mathrm{et} / \mathrm{p} /$ (Mallet 2008 :255, Durand et al. $2011: 124-25$ ). Il y a des résultats mixtes sur le statut des 
consonnes de liaison $/ \mathrm{n} /, \mathrm{z} /$, et $/ \mathrm{t} /$. Les chercheurs qui ont considéré la fréquence des consonnes ont trouvé l'ordre /z/, /n/, /t/, /s/, /p/ (Pagliano et Laks $2005: 5$, Durand et Lyche 2008 :58-59, Durand et al. $2011: 124)$, résultat que Durand et al. (2011:124) qualifient d' " indiscutable » d'après l'analyse de 23953 cas de liaisons. Les différents ordres pour les consonnes de liaison en fonction de leur taux de liaison sont /n/, /z/, /t/ (Malécot $1975: 168$, Mallet $2008: 256$, Ranson $2008: 1675$ ) ou /n/, /t/, /z/ (Ashby 1981 :50, Adda-Decker et al. 2012 :550). Nous pensons qu'il sera plus révélateur de considérer les consonnes en fonction de la classe grammaticale des mots en liaison pour voir quelles consonnes sont employées dans les liaisons variables souvent réalisées et lesquelles dans les liaisons variables rarement réalisées.

En ce qui concerne le sexe des locuteurs, nous prédisons qu'il n'y aura pas de différence significative entre le taux de liaison des femmes et des hommes. La plupart des études antérieures sont arrivées à cette conclusion. Bergen (2005) a conclu que le sexe n'était pas statistiquement significatif chez ses locuteurs suisses et Ranson (2008: 1677) a trouvé un taux de liaison légèrement plus élevé pour les femmes du sud par rapport aux hommes. Cependant, après avoir exclu les sites de liaison variable en théorie mais catégorique dans son corpus, elle a remarqué que le taux de liaison était presque identique pour les hommes et les femmes. L'étude de Durand et al. (2011 :128) a confirmé cette conclusion en montrant qu'il n'y avait pas de différence statistiquement significative entre les deux sexes pour les locuteurs du corpus PFC. Seulement Malécot (1975:169) et Meinschaefer et al. (2015:388) ont noté un plus haut taux de liaison variable chez les femmes que chez les hommes, avec des taux de liaison de $67 \%$ contre $62 \%$ et $36 \%$ contre $21 \%$, respectivement. Dans certains contextes après quand, souvent et depuis dans une lecture et une liste de mots, Booij et De Jong (1987:1017) ont trouvé le même résultat. Ashby (1981:54), par contre, est arrivé au résultat contraire pour ses locuteurs de Tours ; les locutrices ont réalisé $32 \%$ des liaisons contre $36 \%$ chez les locuteurs. Walhout $(2017: 34)$ a trouvé un plus haut taux de liaison chez les hommes dans une ville africaine, Chlef, mais chez les femmes dans deux autres villes, Abijan et Bangui. Puisque les différences de taux de liaison entre les hommes et les femmes étaient minimales, l'auteur a conclu que le sexe était moins influent que les facteurs linguistiques. Malgré notre prédiction que le sexe n'aura pas d'effet significatif sur la réalisation de la liaison, nous allons montrer les résultats en fonction du sexe et de l'âge des locuteurs pour tester cette idée.

\section{Méthodes}

\subsection{Corpus}

Dans le but de bien décrire l'état actuel de la liaison en français parlé, nous avons analysé la parole de 40 locuteurs natifs du français. Vingt de ces locuteurs viennent du nord de la France et vingt du sud. Dans chaque région il y a cinq locuteurs dans chacun des quatre groupes sociaux, organisés selon l'âge et le sexe du locuteur, notamment cinq jeunes femmes, cinq jeunes hommes, cinq femmes âgées, et cinq hommes âgés, comme le montre le Tableau 1.

Les données pour cette étude viennent de deux corpus. Les conversations de tous les locuteurs du nord et trois locuteurs du sud viennent du projet Phonologie du français contemporain (PFC) (Durand et al. 2002). Le projet PFC fournit des transcriptions et enregistrements des conversations entre un intervieweur ou une intervieweuse et un sujet parlant, désignées comme des conversations guidées, et des conversations entre un sujet parlant et ses connaissances, désignées comme des conversations libres. Les conversations analysées dans cette étude ont été enregistrées 
Tableau 1 : Caractéristiques sociales des sujets parlants et taux de liaison

\begin{tabular}{|c|c|c|c|c|c|}
\hline \multicolumn{3}{|l|}{ nord } & \multicolumn{3}{|l|}{ sud } \\
\hline identifiant & $\begin{array}{l}\text { région d'origine } \\
\text { (ville) }\end{array}$ & $\begin{array}{l}\text { taux de } \\
\text { réalisation } \\
\text { (N) }\end{array}$ & identifiant & $\begin{array}{l}\text { région } \\
\text { d'origine } \\
\text { (ville) }\end{array}$ & $\begin{array}{l}\text { taux de } \\
\text { réalisation } \\
\text { (N) }\end{array}$ \\
\hline \multicolumn{6}{|c|}{ jeunes femmes } \\
\hline $\begin{array}{l}\text { NF16 } \\
\text { (50aad1) }\end{array}$ & $\begin{array}{l}\text { Normandie } \\
\text { (Avranches) }\end{array}$ & $\begin{array}{l}8,2 \% \\
(4 / 49)\end{array}$ & SF22 & $\begin{array}{l}\text { PACA } \\
\text { (Rognes) }\end{array}$ & $\begin{array}{l}10.9 \% \\
(6 / 55)\end{array}$ \\
\hline $\begin{array}{l}\text { NF20a } \\
(50 a e v 1)\end{array}$ & $\begin{array}{l}\text { Normandie } \\
\text { (Brécey) }\end{array}$ & $\begin{array}{l}20,8 \% \\
(11 / 53)\end{array}$ & SF23 & $\begin{array}{l}\text { Occitanie } \\
\text { (Alès) }\end{array}$ & $\begin{array}{l}42,9 \% \\
(15 / 35)\end{array}$ \\
\hline $\begin{array}{l}\text { NF20b } \\
\text { (61acg1) }\end{array}$ & $\begin{array}{l}\text { Normandie } \\
\text { (Domfront) }\end{array}$ & $\begin{array}{l}12,3 \% \\
(7 / 57)\end{array}$ & SF25a & $\begin{array}{l}\text { Occitanie } \\
\text { (Mende) }\end{array}$ & $\begin{array}{l}15,7 \% \\
(13 / 83) \\
\end{array}$ \\
\hline $\begin{array}{l}\text { NF25 } \\
\text { (92app1) }\end{array}$ & $\begin{array}{l}\text { Île-de-France } \\
\text { (Paris) }\end{array}$ & $\begin{array}{l}8,1 \% \\
(3 / 37)\end{array}$ & SF25b & $\begin{array}{l}\text { Occitanie } \\
\text { (Nîmes) }\end{array}$ & $\begin{array}{l}23,6 \% \\
(13 / 55) \\
\end{array}$ \\
\hline $\begin{array}{l}\text { NF27 } \\
\text { (92aaf3) }\end{array}$ & $\begin{array}{l}\text { Hauts-de-France } \\
\text { (Roubaix) }\end{array}$ & $\begin{array}{l}31,4 \% \\
(16 / 51)\end{array}$ & SF32 & $\begin{array}{l}\text { Occitanie } \\
\text { (Béziers) }\end{array}$ & $\begin{array}{l}28,6 \% \\
(18 / 63) \\
\end{array}$ \\
\hline \multicolumn{6}{|c|}{ jeunes hommes } \\
\hline $\begin{array}{l}\text { NM17 } \\
(61 \mathrm{acm} 1)\end{array}$ & $\begin{array}{l}\text { Normandie } \\
\text { (Domfront) }\end{array}$ & $\begin{array}{l}6,7 \% \\
(4 / 60) \\
\end{array}$ & SM24 & $\begin{array}{l}\text { PACA } \\
\text { (Martigues) }\end{array}$ & $\begin{array}{l}20,7 \% \\
(19 / 92) \\
\end{array}$ \\
\hline $\begin{array}{l}\text { NM19 } \\
\text { (50ajp1) }\end{array}$ & $\begin{array}{l}\text { Normandie } \\
\text { (Mortagne-au- } \\
\text { Perche) }\end{array}$ & $\begin{array}{l}11,1 \% \\
(6 / 54)\end{array}$ & SM25 & $\begin{array}{l}\text { Occitanie } \\
\text { (Montpellier) }\end{array}$ & $\begin{array}{l}14,3 \% \\
(10 / 70)\end{array}$ \\
\hline $\begin{array}{l}\text { NM24 } \\
\text { (92acj1) }\end{array}$ & $\begin{array}{l}\text { Île-de-France } \\
\text { (Suresnes) }\end{array}$ & $\begin{array}{l}35,9 \% \\
(14 / 39)\end{array}$ & SM26 & $\begin{array}{l}\text { PACA } \\
\text { (Rognes) }\end{array}$ & $\begin{array}{l}13,5 \% \\
(10 / 74)\end{array}$ \\
\hline $\begin{array}{l}\text { NM25 } \\
\text { (92acd1) }\end{array}$ & $\begin{array}{l}\text { Île-de-France } \\
\text { (Issy-les- } \\
\text { Moulineaux) }\end{array}$ & $\begin{array}{l}16,2 \% \\
(6 / 37)\end{array}$ & SM28 & $\begin{array}{l}\text { Occitanie } \\
\text { (Narbonne) }\end{array}$ & $\begin{array}{l}20,7 \% \\
(17 / 82)\end{array}$ \\
\hline $\begin{array}{l}\text { NM31 } \\
\text { (54bfl1) }\end{array}$ & $\begin{array}{l}\text { Grand Est } \\
\text { (Lunéville) }\end{array}$ & $\begin{array}{l}15,4 \% \\
(6 / 39)\end{array}$ & $\begin{array}{l}\text { SM30 } \\
\text { (13bfa1) }\end{array}$ & $\begin{array}{l}\text { PACA (Aix- } \\
\text { en-Provence) }\end{array}$ & $\begin{array}{l}9,4 \% \\
(3 / 29)\end{array}$ \\
\hline \multicolumn{6}{|c|}{ femmes âgées } \\
\hline $\begin{array}{l}\text { NF42 } \\
\text { (50atv1) }\end{array}$ & $\begin{array}{l}\text { Normandie } \\
\text { (Brécey) }\end{array}$ & $\begin{array}{l}20,8 \% \\
(10 / 48)\end{array}$ & SF40 & $\begin{array}{l}\text { PACA } \\
\text { (Avignon) }\end{array}$ & $\begin{array}{l}29,3 \% \\
(29 / 99) \\
\end{array}$ \\
\hline $\begin{array}{l}\text { NF44 } \\
\text { (61asg1) }\end{array}$ & $\begin{array}{l}\text { Normandie } \\
\text { (St. Gilles-des- } \\
\text { Marais) }\end{array}$ & $\begin{array}{l}6,9 \% \\
(4 / 58)\end{array}$ & SF53 & $\begin{array}{l}\text { PACA } \\
\text { (Cucuron) }\end{array}$ & $\begin{array}{l}22,8 \% \\
(13 / 57)\end{array}$ \\
\hline $\begin{array}{l}\text { NF48 } \\
\text { (61ajh1) }\end{array}$ & $\begin{array}{l}\text { Normandie } \\
\text { (Domfront) }\end{array}$ & $\begin{array}{l}23,7 \% \\
(14 / 59)\end{array}$ & SF55 & $\begin{array}{l}\text { Occitanie } \\
\text { (Narbonne) }\end{array}$ & $\begin{array}{l}40,9 \% \\
(36 / 88) \\
\end{array}$ \\
\hline $\begin{array}{l}\text { NF55 } \\
\text { (50alb1) }\end{array}$ & $\begin{array}{l}\text { Normandie } \\
\text { (Brécey) }\end{array}$ & $\begin{array}{l}13,8 \% \\
(8 / 58)\end{array}$ & SF56 & $\begin{array}{l}\text { Occitanie } \\
\text { (Montpellier) }\end{array}$ & $\begin{array}{l}28,7 \% \\
(31 / 108)\end{array}$ \\
\hline $\begin{array}{l}\text { NF69 } \\
\text { (50app1) }\end{array}$ & $\begin{array}{l}\text { Normandie } \\
\text { (Brécey) }\end{array}$ & $\begin{array}{l}20,3 \% \\
(12 / 59)\end{array}$ & $\begin{array}{l}\text { SF57 } \\
(13 b j c 1)\end{array}$ & $\begin{array}{l}\text { PACA (Aix- } \\
\text { en-Provence) }\end{array}$ & $\begin{array}{l}32,8 \% \\
(19 / 58) \\
\end{array}$ \\
\hline \multicolumn{6}{|c|}{ hommes âgés } \\
\hline $\begin{array}{l}\text { NM42 } \\
(50 a p b 1)\end{array}$ & $\begin{array}{l}\text { Normandie } \\
\text { (Brécey) }\end{array}$ & $\begin{array}{l}29,8 \% \\
(17 / 57)\end{array}$ & $\begin{array}{l}\text { SM45 } \\
\text { (13brp2) }\end{array}$ & $\begin{array}{l}\text { PACA } \\
\text { (Marseille) }\end{array}$ & $\begin{array}{l}32,8 \% \\
(19 / 58) \\
\end{array}$ \\
\hline $\begin{array}{l}\text { NM46 } \\
\text { (61adl1) }\end{array}$ & $\begin{array}{l}\text { Normandie } \\
\text { (Céaucé) }\end{array}$ & $\begin{array}{l}9,3 \% \\
(5 / 54)\end{array}$ & SM55a & $\begin{array}{l}\text { PACA } \\
\text { (Rognes) }\end{array}$ & $\begin{array}{l}27,7 \% \\
(31 / 112)\end{array}$ \\
\hline $\begin{array}{l}\text { NM51a } \\
\text { (54bjc1) }\end{array}$ & $\begin{array}{l}\text { Grand Est } \\
\text { (Ogéviller) }\end{array}$ & $\begin{array}{l}18,8 \% \\
(6 / 32)\end{array}$ & SM55b & $\begin{array}{l}\text { PACA } \\
\text { (Marseille) }\end{array}$ & $\begin{array}{l}24,7 \% \\
(19 / 77)\end{array}$ \\
\hline $\begin{array}{l}\text { NM51b } \\
(61 \mathrm{abm} 1)\end{array}$ & $\begin{array}{l}\text { Normandie } \\
\text { (Céaucé) }\end{array}$ & $\begin{array}{l}6,8 \% \\
(4 / 59)\end{array}$ & SM56 & $\begin{array}{l}\text { Occitanie } \\
\text { (Béziers) }\end{array}$ & $\begin{array}{l}21,2 \% \\
(21 / 99)\end{array}$ \\
\hline $\begin{array}{l}\text { NM53 } \\
\text { (50ajm1) }\end{array}$ & $\begin{array}{l}\text { Normandie } \\
\text { (Brécey) }\end{array}$ & $\begin{array}{l}15,8 \% \\
(9 / 57) \\
\end{array}$ & SM65 & $\begin{array}{l}\text { Occitanie } \\
\text { (Conques) }\end{array}$ & $\begin{array}{l}44,3 \% \\
(39 / 88) \\
\end{array}$ \\
\hline
\end{tabular}

entre 2004 et 2006. Les données pour les autres locuteurs du sud viennent du Corpus Montpellier-Rognes qui consiste des conversations enregistrées en 2005 à Montpellier et en 2006 à Rognes par la deuxième auteure qui a déjà analysé les liaisons de 16 de 
ces locuteurs (Ranson 2008). Ces conversations entre un locuteur ou une locutrice et une intervieweuse ressemblent aux conversations guidées du projet PFC, mais elles ont suivi la structure d'une conversation spontanée portant sur les intérêts des locuteurs. Pour bien comparer ces liaisons avec celles des locuteurs du nord, nous avons rajouté les données de 3 locuteurs du projet PFC pour arriver à 5 locuteurs dans chaque groupe social.

Nous avons noté les liaisons variables dans ces conversations sans faire aucune distinction entre les conversations guidées et libres. Nous avons cru que ce choix était justifié par le manque de différence significative entre le taux de liaison dans ces deux situations chez les locuteurs dans 14 enquêtes du projet PFC du nord de la France (Durand et Lyche 2016 :374). Pourtant il peut y avoir des différences de taux de liaison entre les deux sortes de conversation pour des éléments spécifiques, comme est et c'est, comme le montrent Eychenne et al. (2014). Il restera donc à vérifier ces possibles différences dans l'avenir. Le projet PFC fournit aussi la lecture d'une liste de mots et d'un texte par les sujets parlants, mais ces données ne sont pas prises en compte dans la présente étude. En ce qui concerne l'âge des locuteurs, les jeunes du nord ont entre 16 et 31 ans avec un âge moyen de 22 ans, tandis que les jeunes du sud ont entre 22 et 32 ans avec un âge moyen de 26 ans. Les locuteurs âgés du nord ont entre 42 et 69 ans avec un âge moyen de 50 ans et ceux du sud ont entre 40 et 65 ans avec un âge moyen de 54 ans. Les locuteurs du sud ont donc un âge moyen légèrement supérieur à celui des locuteurs du nord.

Les locuteurs du sud viennent de deux provinces; 10 d'Occitanie et 10 de Provence-Alpes-Côte d'Azur (PACA). Leurs villes d'origine en allant de l'ouest vers l'est sont Conques, Mende, Alès, Nîmes, Montpellier, Béziers et Narbonne en Occitanie et Avignon, Cucuron, Rognes, Aix-en-Provence, Martigues et Marseille en Provence-Alpes-Côte d'Azur. Leurs homologues du nord viennent de quatre régions : 14 de Normandie, 3 d'Île-de-France, 1 de Hauts-de-France, et 2 de Grand Est. Leurs villes d'origine comprennent Avranches, Brécey, Saint-Gilles-des-Marais, Domfront en Poiraie, Ceaucé, et Mortagne-au-Perche en Normandie, Suresnes, Issy-lesMoulineaux, et Paris en Île de France, Roubaix en Hauts-de-France, et Lunéville et Ogéviller en Grand Est. Plus de régions sont représentées par les locuteurs du nord afin d'avoir un nombre égal de locuteurs pour chaque groupe social. Vu la distance entre les villes d'origine des locuteurs du nord et entre ceux du sud, nous présentons aussi les taux de liaison des locuteurs individuels dans le Tableau 1. Il ne semble pas y avoir de corrélation entre la ville ou la région d'origine et le taux de liaison chez les locuteurs du nord. Les plus hauts taux de liaison se trouvent chez un jeune locuteur d'Île de France (NM24), une jeune locutrice de Hauts-de-France (NF27) et un homme âgé de Normandie (NM42). Dans le sud, par contre, les plus hauts taux de liaison sont affichés par des locuteurs d'Occitanie et les dix locuteurs d'Occitanie ont un taux de liaison moyen de $28 \%$ contre $22 \%$ chez les locuteurs de Provence-Alpes-Côte d'Azur.

\subsection{Analyse}

Pour calculer le taux de liaison des locuteurs, nous avons d'abord noté tous les sites possibles de liaison variable sur la transcription écrite des conversations. Ces sites sont des endroits où un mot se terminant par une consonne latente est suivi par un mot commençant par une voyelle selon les fonctions grammaticales des deux mots. Ces sites de liaison, identifiés par Delattre $(1966: 43)$ et par la suite modifiés par Encrevé (1988:47), Valdman (1993:150-76), Lodge et al. (1997:99), et Armstrong (2001 :182), comprennent un nom pluriel suivi d'un adjectif (étudiants étrangers) ou d'un verbe (les ânes étaient passés), un verbe suivi d'une autre forme verbale (ils sont allés, ils peuvent être) ou d'un complément (j'entretiens un peu), et certains mots 
invariables, comme les prépositions après, dans, chez, en, sans, sous, les adverbes beaucoup, bien, puis, plus, moins, mieux, souvent, très, trop, et les adverbes en -ment, les particules négatives pas et jamais, et les conjonctions mais et quand. Nous avons ajouté à cette liste l'expression c'est-à-dire, considérée auparavant comme liaison obligatoire, mais qui est maintenant variable selon Green et Hintze (1990:73) et Ranson $(2008: 1672)$. Après avoir trouvé tous les sites possibles de liaison variable, nous avons écouté les enregistrements et noté si les locuteurs avaient fait chaque liaison ou non. Nous avons choisi de vérifier les liaisons déjà codées dans le projet PFC pour être sûres d'analyser les mêmes liaisons dans les deux régions. Les sites de liaison interrompus par une pause ou euh et ceux où il était impossible d'entendre la consonne de liaison en raison de la parole simultanée ou des bruits de fond ont été exclus.

Nous avons trouvé un minimum de 29 sites de liaison chez chaque locuteur et un maximum de 60 sites de liaison chez les locuteurs du nord dans 10 minutes de conversation. Chez les locuteurs du sud, nous avons identifié tous les sites de liaison dans 10 minutes de conversation, jusqu'à 112 par locuteur. ${ }^{2}$ Nous avons inclus tous les sites de liaison variable dans nos premiers calculs du taux de liaison chez les locuteurs des deux régions dans les Tableaux 2 et 3 ci-dessous. Dans les analyses subséquentes, nous avons raffiné nos calculs en excluant les liaisons qui n'étaient pas variables dans au moins un des corpus, comme nous verrons dans le Tableau 3. Les sites de liaison exclus sont entre nom pluriel et adjectif ou verbe, après avoir auxiliaire, après d'autres verbes, après les adverbes polysyllabiques ailleurs, alors, assez, beaucoup, maintenant, surtout et les adverbes en -ment, les prépositions après, avant et devant, la conjonction mais et l'adverbe puis, et les adverbes négatifs pas et jamais.

\section{Résultats et discussion}

Nous considérons d'abord les facteurs diastratiques pour voir si, de façon générale, les jeunes font moins souvent la liaison que les personnes âgées. En présentant séparément les taux d'élision selon la région d'origine du locuteur, nous pourrons savoir si les locuteurs septentrionaux font moins de liaisons que leurs homologues méridionaux et s'il y a un plus grand écart entre le taux de liaison des locuteurs septentrionaux et méridionaux plus âgés qu'entre leurs homologues plus jeunes.

\subsection{Facteurs diastratiques}

Le Tableau 2 présente les taux de liaison en fonction de l'âge, du sexe et du lieu d'origine des locuteurs. Pour calculer le taux de liaison, nous avons divisé le nombre de liaisons variables faites par chaque groupe de locuteurs par le nombre de liaisons possibles. Le tableau présente les résultats pour les locuteurs regroupés selon l'âge et le sexe dans les premières rangées et ensuite selon leur groupe social qui combine les caractéristiques de l'âge et du sexe en quatre groupes. Pour savoir si l'effet d'un facteur diastratique est significatif, ce tableau présente aussi les valeurs $p$ d'un test du chi carré. Les cases ombrées indiquent les valeurs qui sont significatives si on établit le seuil de significativité à moins de 0,05.

Le taux de liaison réalisée, représenté par les pourcentages dans le Tableau 2, montre que le taux de liaison est toujours plus élevé chez les locuteurs du sud, quel que soit l'âge ou le sexe des locuteurs. Cependant, le test du chi carré indique que la région n'est pas significative chez les jeunes locuteurs. L'âge du locuteur est significatif seulement dans le sud et le sexe n'est jamais significatif. En somme, ce sont les locuteurs âgés du sud, femmes et hommes, qui font le plus souvent la liaison. 
La Figure 1, qui montre les résultats selon le groupe social des locuteurs, permet de visualiser l'effet des caractéristiques des locuteurs sur la réalisation de la liaison variable.

Tableau 2 : Taux de liaison variable réalisée en fonction de l'âge, du sexe, et de la région d'origine du locuteur

\begin{tabular}{|l|l|l|l|l|l|}
\hline & nord $(\mathrm{N})$ & sud $(\mathrm{S})$ & \multicolumn{3}{l|}{$\begin{array}{l}\text { Significativité par le test du chi } \\
\text { carré }: \text { valeur } p \text { (significatif) }\end{array}$} \\
\hline & & & région & âge & sexe \\
\hline jeunes (J) & $16,0 \%(76 / 474)$ & $19,3 \%(124 / 641)$ & 0,234 & N $0,870^{3}$ & \\
\hline âgés (A) & $16,5 \%(89 / 540)$ & $30,5 \%(257 / 843)$ & 0,000 & S 0,000 & \\
\hline femmes (F) & $16,7 \%(88 / 527)$ & $27,5 \%(193 / 701)$ & 0,000 & & N 0,746 \\
\hline hommes (H) & $15,8 \%(77 / 487)$ & $24,0 \%(188 / 783)$ & 0,004 & & S 0,233 \\
\hline \hline $\begin{array}{l}\text { jeunes } \\
\text { femmes }\end{array}$ & $16,3 \%(40 / 246)$ & $22,3 \%(65 / 291)$ & 0,146 & NF 0,831 & NJ 0,906 \\
\hline $\begin{array}{l}\text { jeunes } \\
\text { hommes }\end{array}$ & $15,8 \%(36 / 228)$ & $16,9 \%(59 / 350)$ & 0,774 & NH 0,992 & SJ 0,151 \\
\hline femmes âgées & $17,1 \%(48 / 281)$ & $31,2 \%(128 / 410)$ & 0,001 & SF 0,049 & NA0,740 \\
\hline hommes âgés & $15,8 \%(41 / 259)$ & $29,8 \%(129 / 433)$ & 0,001 & SH 0,001 & SA0,743 \\
\hline total & $\begin{array}{l}16,3 \% \\
(165 / 1014)\end{array}$ & $25,7 \%(381 / 1484)$ & 0,000 & & \\
\hline
\end{tabular}

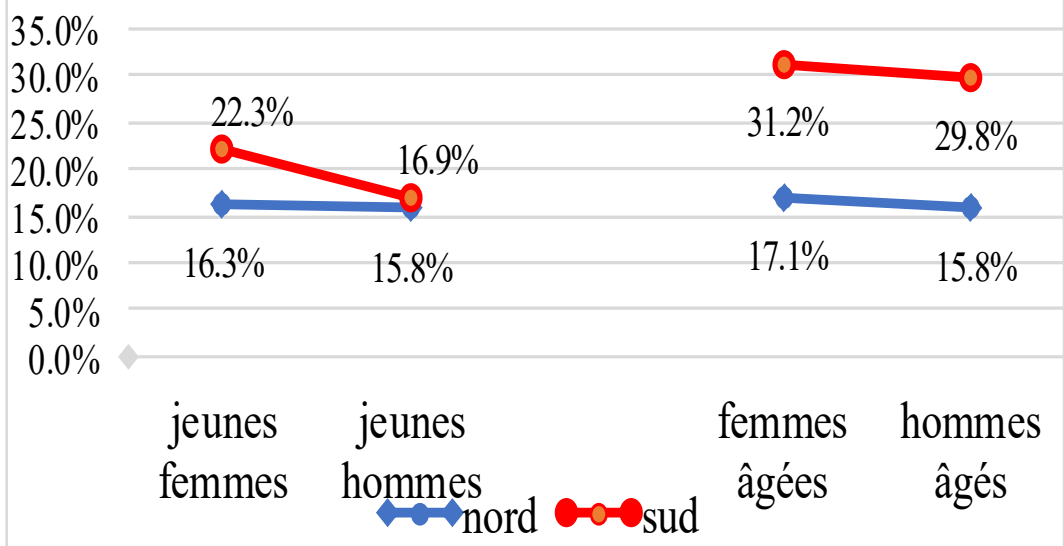

Fig. 1 : Réalisation de la liaison variable en fonction de l'âge et de l'origine des locuteurs

Ces résultats permettent de revenir sur nos trois hypothèses concernant les caractéristiques des locuteurs. La première hypothèse que les locuteurs plus âgés font plus souvent la liaison est validée seulement pour les locuteurs du sud. Chez les locuteurs du nord, cependant, le taux de liaison est le même chez les jeunes hommes et les âgés $(15,8 \%)$ et légèrement plus élevé chez les femmes âgées que chez les jeunes femmes $(17,1 \%$ vs. $16,3 \%)$, mais cette différence n'est pas significative. Une comparaison en fonction de la région d'origine montre que le taux de liaison variable est plus élevé chez les locuteurs du sud que chez ceux du nord (25,7\% contre $16,3 \%)$ pour tous les groupes sociaux, comme l'avait prédit la deuxième hypothèse. Pourtant, cette différence diatopique est significative seulement chez les locuteurs plus âgés. Finalement, nous constatons qu'il y a moins de différence entre les taux de liaison des jeunes locuteurs qu'entre ceux des locuteurs plus âgés. La plus petite différence entre les régions est chez les jeunes hommes (un écart de 1,1) suivi par les jeunes femmes (un écart de 6,0). Chez les locuteurs âgés la différence est de 14,1 chez les femmes 
âgées et de 14,0 chez les hommes âgés. Il est clair que les liaisons variables se réalisent moins chez les locuteurs du nord et chez les jeunes du sud, ce qui efface la différence régionale pour le taux de liaison chez les jeunes. Pour mieux décrire le statut de la liaison en français parlé, nous verrons l'effet de la classe grammaticale du mot liaisonnant et de la consonne de liaison dans la section suivante.

\subsection{Facteurs linguistiques}

Une analyse de l'effet de la classe grammaticale des mots en liaison et la consonne de liaison montre le statut de la liaison de certaines consonnes dans certains contextes syntaxiques. Le Tableau 3 montre le taux de liaison en fonction de la classe grammaticale du mot liaisonnant et de la région d'origine des locuteurs. La colonne à gauche regroupe les classes grammaticales du mot liaisonnant, suivi par un exemple de cette sorte de liaison réalisée par un locuteur de notre corpus en liaisons variables fréquentes, liaisons variables vraiment variables, et liaisons variables rares. Les chiffres en caractères gras montrent la région avec le plus haut taux de liaison pour chaque classe grammaticale.

Tableau 3 : Taux de liaison variable réalisée en fonction de la région et de la catégorie grammaticale du mot liaisonnant

\begin{tabular}{|c|c|c|}
\hline & nord & sud \\
\hline \multicolumn{3}{|l|}{ liaisons souvent réalisées } \\
\hline $\begin{array}{l}\text { adverbe très } \\
\text { très intéressant }\end{array}$ & $100 \%(7 / 7)$ & $97,5 \%(39 / 40)$ \\
\hline $\begin{array}{l}\text { prépositions : sans, en, dans, chez } \\
\text { sans arrêt }\end{array}$ & $88,4 \%(76 / 86)$ & $\mathbf{9 4 , 2 \%}(98 / 104)$ \\
\hline nombre de liaisons fréquentes non réalisées & 10 & 7 \\
\hline \multicolumn{3}{|l|}{ liaisons variablement réalisées } \\
\hline $\begin{array}{l}\text { conjonction quand } \\
\text { quand on est dehors }\end{array}$ & $75 \%(33 / 44)$ & $72,6 \%(45 / 62)$ \\
\hline $\begin{array}{l}\text { adverbes bien et moins } \\
\text { bien organisé, moins on supporte }\end{array}$ & $\mathbf{7 0 , 6 \%}(12 / 17)$ & $50 \%(8 / 16)$ \\
\hline $\begin{array}{l}\text { être (auxiliaire) présent de l'indicatif 3sg } \\
\text { on est allé }\end{array}$ & $63,6 \%(7 / 11)$ & $\mathbf{8 6 , 7 \%}(26 / 30)$ \\
\hline c'est-à-dire & $57,1 \%(4 / 7)$ & $44,4 \%(8 / 18)$ \\
\hline $\begin{array}{l}\text { être (auxiliaire) autres temps et personnes } \\
\text { je suis allée }\end{array}$ & $15 \%(6 / 40)$ & $\mathbf{5 4 , 1 \%}(20 / 37)$ \\
\hline $\begin{array}{l}\text { être (copule) présent de l'indicatif 3sg } \\
\text { c'est un endroit }\end{array}$ & $13,9 \%(17 / 122)$ & $\mathbf{3 3 , 5 \%}(105 / 313)$ \\
\hline \multicolumn{3}{|l|}{ liaisons rarement réalisées } \\
\hline $\begin{array}{l}\text { trop } \\
\text { trop abîmés, trop étroite }\end{array}$ & $16,7 \%(1 / 6)$ & $12,5 \%(1 / 8)$ \\
\hline $\begin{array}{l}\text { être (copule) autres personnes } \\
\text { je suis étudiant }\end{array}$ & $1,8 \%(2 / 113)$ & $\mathbf{8 , 9 \%}(10 / 112)$ \\
\hline $\begin{array}{l}\text { nom pl. + adj. / verbe } \\
\text { personnes âgées }\end{array}$ & $0 \%(0 / 14)$ & $11,9 \%(7 / 59)$ \\
\hline $\begin{array}{l}\text { avoir (auxiliaire) } \\
\text { ils avaient été scolarisés }\end{array}$ & $0 \%(0 / 36)$ & $7,9 \%(3 / 38)$ \\
\hline $\begin{array}{l}\text { autres verbes } \\
\text { il y avait un animal }\end{array}$ & $0 \%(0 / 242)$ & $\mathbf{2 , 7 \%}(9 / 329)$ \\
\hline $\begin{array}{l}\text { pas et jamais } \\
\text { pas un seul instant }\end{array}$ & $0 \%(0 / 88)$ & $1,1 \%(1 / 95)$ \\
\hline $\begin{array}{l}\text { alors, après, avant, mais, puis } \\
\text { après avoir fait ça }\end{array}$ & $0 \%(0 / 138)$ & $\mathbf{0 , 6 \%}(1 / 159)$ \\
\hline
\end{tabular}




\begin{tabular}{|l|l|l|}
\hline $\begin{array}{l}\text { adverbe polysyllabique : assez, beaucoup, } \\
\text { maintenant, souvent, surtout, adverbes en } \\
\text {-ment }\end{array}$ & $0 \%(0 / 43)$ & $0 \%(0 / 64)$ \\
\hline
\end{tabular}

On constate que le taux de réalisation de presque toutes les liaisons rares est plus élevé dans le sud, mais ce n'est pas le cas pour toutes les liaisons fréquentes et variables. Parmi les liaisons fréquentes, la liaison est toujours réalisée après l'adverbe très à une exception près. Une locutrice du sud (SF56) a dit c'est très important sans faire la liaison après très. Après les prépositions, les locuteurs du nord n'ont pas réalisé la liaison 5 fois après dans, 3 fois après en, et 2 fois après sans, mais ils ont toujours réalisé la liaison après chez. Les locuteurs du sud n'ont pas réalisé 3 liaisons après dans, 2 après en et une après chez, mais ils ont toujours réalisé la liaison après sans. Le Tableau 4 présente le taux de liaison après les prépositions individuelles. Les exemples des liaisons non réalisées sont présentés pour chaque préposition. Il est intéressant de voir que c'est le même locuteur du nord (NM46) qui est responsable de 5 des 10 liaisons non réalisées. Dans le sud ce sont des locuteurs différents qui ne réalisent pas une occurrence de ces liaisons fréquentes. On pourrait penser que ces liaisons sont en train de devenir invariables, mais le petit nombre de non-réalisations nous fait savoir qu'elles ne sont pas encore arrivées à ce statut. En outre, ces liaisons fréquentes montrent que la réalisation de certaines liaisons n'est pas en train de diminuer.

Tableau 4 : Taux de liaison réalisée après préposition en fonction de la région

\begin{tabular}{|c|c|c|}
\hline & nord & sud \\
\hline \multirow[t]{2}{*}{ sans } & $33,3 \%(1 / 3)$ & $100 \%(7 / 7)$ \\
\hline & $\begin{array}{l}\text { sans avoir la visite (NM46) } \\
\text { sans être formé (NM46) }\end{array}$ & -- \\
\hline \multirow[t]{2}{*}{ en } & $92,7 \%(38 / 41)$ & $95,7 \%(45 / 47)$ \\
\hline & $\begin{array}{l}\text { en électronique ( } 2 \text { fois) (NM31) } \\
\text { confiance en elle (NF42) }\end{array}$ & $\begin{array}{l}\text { en anglais (SF57) } \\
\text { en été (SM25) }\end{array}$ \\
\hline \multirow[t]{2}{*}{ dans } & $87,2 \%(34 / 39)$ & $93,2 \%(41 / 44)$ \\
\hline & $\begin{array}{l}\text { dans un lycée }(\mathrm{NF} 20 \mathrm{~b}) \\
\text { dans un camp }(\mathrm{NM} 46) \\
\text { dans un village }(\mathrm{NM} 46) \\
\text { dans un mas }(\mathrm{NM} 46) \\
\text { dans un très bon établissement } \\
\text { (NM25) }\end{array}$ & $\begin{array}{l}\text { dans une administration (SM56) } \\
\text { dans une famille (SF53) } \\
\text { dans un centre (SF40) }\end{array}$ \\
\hline \multirow[t]{2}{*}{ chez } & $100 \%(3 / 3)$ & $83,3 \%(5 / 6)$ \\
\hline & -- & chez un ami (SM26) \\
\hline
\end{tabular}

Ce sont les liaisons rares plutôt qui montrent la disparition de la consonne de liaison dans certains contextes syntaxiques. Aucun locuteur de nos deux corpus n'a réalisé la liaison après les adverbes polysyllabiques, comme souvent, maintenant et vraiment, et les locuteurs du nord n'ont jamais réalisé la liaison entre un nom pluriel et un adjectif ou verbe, après avoir auxiliaire, après des verbes à part être, après pas et jamais, et après alors, après, avant, mais et puis. En tout, les locuteurs du nord n'ont fait que 3 liaisons rares. Deux de ces liaisons, trop abîmés et ils sont à Ducey, ont été produites par le même locuteur plus âgé (NM42), et la troisième, je suis en maîtrise, par un jeune locuteur (NM24). Chez les locuteurs du sud, ces liaisons sont rares, mais encore possibles chez quelques locuteurs. La liaison après chacun des mots trop, pas et après est réalisée une seule fois dans trop étroite (SF40), pas un seul instant (SM45) et après avoir fait ça (SF55), respectivement. La liaison après avoir auxiliaire est réalisée 3 fois. Pourtant, deux de ces réalisations sont dans la même expression prononcée par la même locutrice (SF55): ils avaient été scolarisés. L'autre réalisation apparaît dans mes premiers étudiants ... ont été des étudiants chiliens (SM65). Pour 
les autres fonctions grammaticales des liaisons rares, la liaison est faite 10 fois après être comme copule dans d'autres temps ou personnes que le présent de l'indicatif au $3 \mathrm{sg}, 9$ fois après d'autres verbes, et 7 fois après un nom pluriel, mais seulement dans les expressions fréquentes personnes âgées (3 fois), étudiants étrangers (3 fois), et affaires étrangères ( 1 fois) qui n'apparaissent pas dans les conversations du nord. On peut donc conclure que certaines liaisons sont pratiquement perdues chez les locuteurs du nord mais peuvent encore se produire chez certains locuteurs du sud.

Parmi les liaisons variablement réalisées, le taux de liaison est similaire après quand pour les locuteurs du nord $(75 \%)$ et du sud $(72,6 \%)$. Les locuteurs du nord affichent un bien plus haut taux de liaison après les adverbes bien et moins (avec un écart de 20,6) et l'expression c'est-à-dire $e^{4}$ (avec un écart de 12,7), mais il y a peu d'occurrences de ces liaisons ; leur nombre ne dépasse jamais 18 dans une région. Les autres liaisons vraiment variables sont après être, notamment après être auxiliaire au $3 \mathrm{sg} \mathrm{du}$ présent de l'indicatif, être auxiliaire à d'autres personnes et temps, et être comme copule au $3 \mathrm{sg}$ du présent de l'indicatif. Ce sont les fonctions grammaticales ayant une différence de plus de 10 pour cent entre les deux régions et avec plus de 20 occurrences dans au moins une région. Puisque ce sont ces liaisons qui permettent de mieux comprendre les différents taux de liaison entre les locuteurs du nord et du sud, nous les analysons en plus grand détail dans le Tableau 5.

Dans ce tableau, et dans la Figure 2 qui permet de mieux visualiser ces résultats, nous voyons clairement que les jeunes locuteurs du nord ne font que rarement ces liaisons qui restent encore variables chez d'autres groupes de locuteurs. Les jeunes femmes n'ont réalisé que 3 de ces liaisons et les jeunes hommes n'en ont fait que 5 . Les locuteurs âgés du nord n'ont pas réalisé beaucoup de ces liaisons non plus, seulement 10 chez les hommes et 12 chez les femmes. Chez les locuteurs du sud, les jeunes les réalisent bien moins souvent que les locuteurs plus âgés, ce qui suggère une diminution de la consonne de liaison dans cette région. En ce qui concerne la liaison selon les différentes fonctions d'être, il y a peu d'occurrences dans le corpus du verbe être comme copule au 3sg suivi par une voyelle, mais la liaison est souvent réalisée dans ce contexte. Il y a aussi peu d'occurrences d'être comme auxiliaire au $3 \mathrm{sg}$ devant voyelle, mais dans ce contexte la liaison n'est pas souvent réalisée, sauf par les hommes du sud âgés et surtout par les femmes âgées de cette région. Le verbe être est employé le plus souvent comme auxiliaire aux autres temps et personnes que $3 \mathrm{sg} d u$ présent de l'indicatif. Les jeunes locuteurs du nord les réalisent le moins souvent, suivis par les locuteurs du nord plus âgés et les jeunes locuteurs du sud. Ce sont seulement les locuteurs du sud âgés qui réalisent plus de la moitié de ces liaisons.

Tableau 5 : Taux de liaison variable réalisée après être selon la région et le groupe social

\begin{tabular}{|c|c|c|c|c|c|c|c|c|}
\hline & \multicolumn{2}{|c|}{ jeunes femmes } & \multicolumn{2}{|c|}{ jeunes hommes } & \multicolumn{2}{|c|}{ femmes âgées } & \multicolumn{2}{|c|}{ hommes âgés } \\
\hline & nord & sud & nord & sud & nord & sud & nord & sud \\
\hline $\begin{array}{l}\text { être aux. } \\
\text { 3sg présent }\end{array}$ & $\begin{array}{l}0 \% \\
(0 / 5)\end{array}$ & $\begin{array}{l}0 \% \\
(0 / 2)\end{array}$ & $\begin{array}{l}7,7 \% \\
(1 / 13)\end{array}$ & $\begin{array}{l}25 \% \\
(1 / 4)\end{array}$ & $\begin{array}{l}26,7 \% \\
(4 / 15)\end{array}$ & $\begin{array}{l}68,2 \% \\
(15 / 22)\end{array}$ & $\begin{array}{l}14,3 \% \\
(1 / 7)\end{array}$ & $\begin{array}{l}44,4 \% \\
(4 / 9)\end{array}$ \\
\hline $\begin{array}{l}\text { être aux. } \\
\text { autres } \\
\text { temps/ } \\
\text { personnes }\end{array}$ & $\begin{array}{l}5,3 \% \\
(2 / 38)\end{array}$ & $\begin{array}{l}19,5 \% \\
(16 / 82)\end{array}$ & $\begin{array}{l}8,6 \% \\
(3 / 35)\end{array}$ & $\begin{array}{l}15,4 \% \\
(12 / 78)\end{array}$ & $\begin{array}{l}15 \% \\
(3 / 20)\end{array}$ & $\begin{array}{l}50 \% \\
(34 / 68)\end{array}$ & $\begin{array}{l}31,0 \% \\
(9 / 29)\end{array}$ & $\begin{array}{l}50,6 \% \\
(43 / 85)\end{array}$ \\
\hline $\begin{array}{l}\text { être copule } \\
3 \text { sg présent }\end{array}$ & $\begin{array}{l}50 \% \\
(1 / 1)\end{array}$ & $\begin{array}{l}75 \% \\
(3 / 4) \\
\end{array}$ & $\begin{array}{l}25 \% \\
(1 / 4)\end{array}$ & $\begin{array}{l}60 \% \\
(3 / 5)\end{array}$ & $\begin{array}{l}100 \% \\
(5 / 5)\end{array}$ & $\begin{array}{l}100 \% \\
(4 / 4)\end{array}$ & -- & $\begin{array}{l}94,1 \% \\
(16 / 17)\end{array}$ \\
\hline total & $\begin{array}{l}6,8 \% \\
(3 / 44)\end{array}$ & $\begin{array}{l}21,6 \% \\
(19 / 88)\end{array}$ & $\begin{array}{l}9,6 \% \\
(5 / 52)\end{array}$ & $\begin{array}{l}18,4 \% \\
(16 / 87)\end{array}$ & $\begin{array}{l}30 \% \\
(12 / 40)\end{array}$ & $\begin{array}{l}56,4 \% \\
(53 / 94)\end{array}$ & $\begin{array}{l}27,8 \% \\
(10 / 36)\end{array}$ & $\begin{array}{l}56,8 \% \\
(63 / 111)\end{array}$ \\
\hline
\end{tabular}




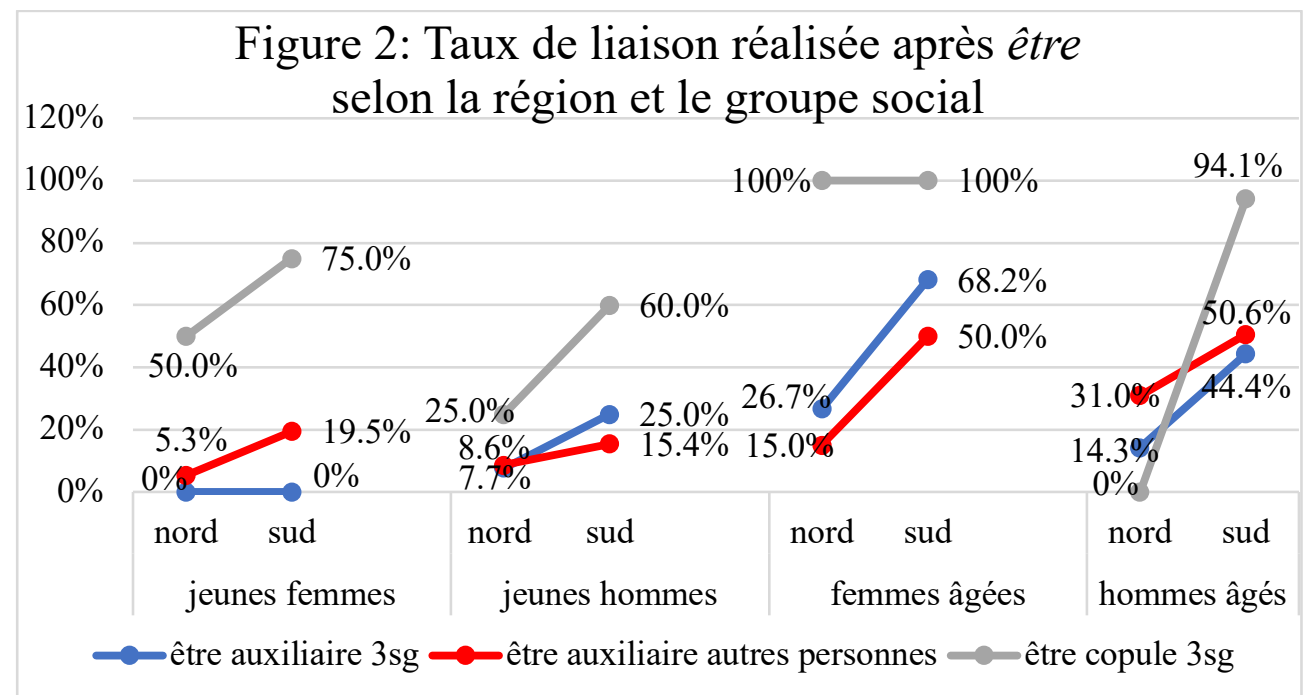

Nous venons de voir que la liaison se fait encore souvent dans certains contextes syntaxiques, comme après l'adverbe très et les prépositions sans, en, dans, chez, qu'elle se fait encore de façon variable dans d'autres contextes, comme après quand, les adverbes bien et moins et le verbe être, et qu'elle ne se fait que rarement après un nom pluriel, les adverbes négatifs pas et jamais, et les mots alors, après, avant, mais, puis, les autres verbes, et qu'elle ne se fait jamais après un adverbe polysyllabique. Nous allons voir maintenant la corrélation entre ces sortes de liaison et la consonne de liaison. Il faut noter tout d'abord que la consonne de liaison /в/ est réalisée si rarement dans les liaisons variables, comme prêter à confusion, que nous n'avons pas noté ces sites de liaison dans le corpus. La consonne /p/ est rare en liaison aussi, prononcée seulement deux fois dans trop abîmés et trop étroite. Les consonnes de liaison qui sont encore fréquentes sont $/ \mathrm{n} /, \mathrm{t} /$, et $/ \mathrm{z} /$. Le Tableau 6 montre le taux de liaison général pour ces consonnes fréquentes et la proportion de ces consonnes dans des sites de liaisons pour les liaisons classées comme fréquentes, vraiment variables, et rares. Les différences significatives entre le nord et le sud $(\mathrm{p}<0,05)$ sont indiquées par des cases ombrées.

Tableau 6 : Taux et proportion des liaisons en fonction de la consonne de liaison, de la région, et de la sorte de liaison selon sa fréquence

\begin{tabular}{|c|c|c|c|c|c|c|}
\hline & \multicolumn{2}{|l|}{$/ \mathrm{n} /$} & \multicolumn{2}{|l|}{$/ \mathrm{t} /$} & \multicolumn{2}{|l|}{$\mid \mathrm{z} /$} \\
\hline & nord & sud & nord & sud & nord & sud \\
\hline taux général & $\begin{array}{l}90,7 \% \\
(49 / 54) \\
\end{array}$ & $\begin{array}{l}85,0 \% \\
(51 / 60) \\
\end{array}$ & $\begin{array}{l}12,2 \% \\
(62 / 509) \\
\end{array}$ & $\begin{array}{l}23,8 \% \\
(202 / 847)\end{array}$ & $\begin{array}{l}12,0 \% \\
(53 / 442) \\
\end{array}$ & $\begin{array}{l}22,4 \% \\
(127 / 566)\end{array}$ \\
\hline $\begin{array}{l}\text { taux de liaison } \\
\text { fréquente }\end{array}$ & $\begin{array}{l}92,7 \% \\
(38 / 41)\end{array}$ & $\begin{array}{l}95,7 \% \\
(45 / 47)\end{array}$ & 0 & 0 & $\begin{array}{l}86,5 \% \\
(45 / 52)\end{array}$ & $\begin{array}{l}95,6 \% \\
(87 / 91)\end{array}$ \\
\hline $\begin{array}{l}\text { taux de liaison } \\
\text { vraiment } \\
\text { variable }\end{array}$ & $\begin{array}{l}84,6 \% \\
(11 / 13)\end{array}$ & $\begin{array}{l}46,2 \% \\
(6 / 13)\end{array}$ & $\begin{array}{l}32,1 \% \\
(61 / 190)\end{array}$ & $\begin{array}{l}38,1 \% \\
(192 / 504)\end{array}$ & $\begin{array}{l}18,4 \% \\
(7 / 38)\end{array}$ & $\begin{array}{l}33,8 \% \\
(24 / 71)\end{array}$ \\
\hline \multicolumn{7}{|c|}{ Proportion des liaisons variables... } \\
\hline fréquentes & $\begin{array}{l}75,9 \% \\
(41 / 54)\end{array}$ & $\begin{array}{l}78,3 \% \\
(47 / 60)\end{array}$ & 0 & 0 & $\begin{array}{l}11,8 \% \\
(52 / 442)\end{array}$ & $\begin{array}{l}16,1 \% \\
(91 / 566)\end{array}$ \\
\hline $\begin{array}{l}\text { vraiment } \\
\text { variables }\end{array}$ & $\begin{array}{l}24,1 \% \\
(13 / 54)\end{array}$ & $\begin{array}{l}21,7 \% \\
(13 / 60)\end{array}$ & $\begin{array}{l}37,3 \% \\
(190 / 509)\end{array}$ & $\begin{array}{l}59,5 \% \\
(504 / 847)\end{array}$ & $\begin{array}{l}8,6 \% \\
(38 / 442)\end{array}$ & $\begin{array}{l}12,5 \% \\
(71 / 566)\end{array}$ \\
\hline rares & 0 & 0 & $\begin{array}{l}62,7 \% \\
(319 / 509)\end{array}$ & $\begin{array}{l}40,5 \% \\
(343 / 847)\end{array}$ & $\begin{array}{l}79,6 \% \\
(352 / 442)\end{array}$ & $\begin{array}{l}71,4 \% \\
(404 / 566)\end{array}$ \\
\hline
\end{tabular}


On constate que $/ \mathrm{n} / \mathrm{se}$ trouve le plus souvent dans des liaisons fréquentes (après la préposition en) et moins souvent dans des liaisons vraiment variables (après bien). Cette répartition explique en grande mesure son haut taux de liaison réalisée. Toutefois, le taux de liaison relativement bas après bien dans le sud indique que cette liaison est peut-être en train de diminuer pendant que la liaison après en reste fréquente. La consonne de liaison /t/ se trouve le plus souvent dans des liaisons rares dans la parole des locuteurs du nord et dans des liaisons vraiment variables dans la parole des locuteurs du sud. Cette différence significative s'explique en grande partie, comme nous pouvons déduire des résultats présentés dans le Tableau 3 , par le plus grand nombre de sites de liaison après être copule $3 \mathrm{sg}$ chez les locuteurs du sud ( $21 \%$ du nombre total de sites de liaison) que chez ceux du nord (11\%). Il est intéressant de noter que le taux pour toutes les liaisons vraiment variables avec $/ \mathrm{t} /$ est similaire dans les deux régions, donc ce qui crée la différence entre le taux de liaison général dans les deux régions est la différente proportion des liaisons vraiment variables plutôt que rares. La consonne de liaison $/ \mathrm{z} / \mathrm{se}$ trouve surtout dans les liaisons rares, mais son taux de liaison est plus élevé dans les liaisons vraiment variables chez les locuteurs du sud que ceux du nord.

Ces résultats suggèrent une tendance des locuteurs à associer la consonne $/ \mathrm{z} / \mathrm{avec}$ la liaison invariable, soit obligatoire soit interdite, et la consonne /t/ avec la liaison variable. Cette tendance est motivée possiblement par le fait que les liaisons obligatoires, comme entre déterminant et nom ou pronom et verbe, se font toujours avec la consonne /z/. Dans notre corpus, la consonne /t/ n'est jamais la consonne de liaison dans les liaisons fréquentes, de la même façon qu'elle n'est jamais la consonne de liaison dans les liaisons obligatoires. Dans les liaisons vraiment variables, la consonne de liaison est /t/ après quand et après est et sont et plus rarement étai(en)t. Les seules liaisons vraiment variables avec la consonne /z/ sont après moins et après suis, es, sommes, êtes et étais. Nous constatons donc une différence entre les consonnes de liaison /z/ dans les liaisons variables fréquentes ou rares et / $t$ / dans les liaisons vraiment variables.

\section{Conclusion}

L'objectif de cette étude était de voir s'il existe des différences en fonction de l'âge, du sexe et de la région d'origine des locuteurs et de la classe grammaticale du mot liaisonnant ou de la consonne de liaison pour les liaisons variables. Nos résultats montrent que les jeunes du sud de la France réalisent moins souvent la liaison que leurs homologues âgés, ce qui suggère, mais ne confirme pas, un changement en cours vers la perte de la consonne finale dans cette région. Cependant, cette différence ne se manifeste pas au nord où il n'y a presque pas de différence entre les jeunes et les âgés. Quel que soit l'âge des locuteurs du sud, ils réalisent la liaison plus souvent que ceux du nord. Le fait que les locuteurs du nord et les jeunes du sud réalisent la liaison moins souvent réduit la différence régionale chez les jeunes. Nous avons classé les liaisons variables selon celles qui sont fréquentes, rares et vraiment variables. Quant aux liaisons fréquentes, elles pourraient être en voie de devenir invariables vu le taux très haut de leur réalisation. En plus, cela indique que la réalisation de certaines liaisons ne diminue pas. Parmi les liaisons rares certaines semblent avoir disparu, tandis que d'autres se produisent encore au sud mais à un taux très faible. Nous avons identifié six liaisons vraiment variables dont trois montrent la plus grande différence entre les régions et qui ont plus de vingt occurrences. Ces liaisons, être auxiliaire 3sg, être auxiliaire à d'autres personnes et temps et être copule $3 \mathrm{sg}$, font preuve d'un taux de liaison plus haut chez les locuteurs du sud à deux exceptions où les locuteurs des deux régions réalisent la liaison à un taux égal. Nous pouvons conclure que la liaison est en diminution chez les jeunes locuteurs du sud et dans beaucoup de contextes de liaison. 


\section{Références bibliographiques}

Ashby, W. J. (1981). French liaison as a sociolinguistic phenomenon. Linguistic symposium on Romance Languages, éd. par W. W. Cressey et D. J. Napoli, 46-57. Washington, DC : Georgetown University Press.

Armstrong, N. (2001). Social and Stylistic Variation in Spoken French: A comparative approach. Amsterdam/Philadelphia : John Benjamins.

Bergen, Benjamin K. (2005). Social Variability and Probabilistic Language Processing. International Computer Science Institute Technical Report. http://www.cogsci.ucsd.edu/ $\sim$ bkbergen/papers/cogsci02.pdf.

Booij, G. et De Jong, D. (1987). The domain of liaison: Theories and data. Linguistics, 25, 1005-25.

Delattre, P. (1966). Studies in French and Comparative Phonetics. La Haye : Mouton.

Durand, J., Laks, B., Calderone B., et Tchobanov, A. (2011). Que savons-nous de la liaison aujourd'hui ? Langue française, 169, 103-35.

Durand, J., Laks, B., et Lyche, C. (2002). La phonologie du français contemporain: usages, variétés et structure. Romanistische Korpuslinguistik- Korpora und gesprochene Sprache/Romance Corpus Linguistics - Corpora and Spoken Language, éd par C. Pusch et W. Raible, 93-106. Tübingen : Gunter Narr.

Durand, J. et Lyche, C. (2008). French liaison in the light of corpus data. French Language Studies, 18, 33-66.

Durand, J. et Lyche, C. (2016). Approaching variation in PFC: The liaison level. Varieties of Spoken French, éd. par S. Detey, J. Durand, B. Laks, et C. Lyche, 363-75. Oxford: Oxford University Press.

Encrevé, P. (1988). La liaison avec et sans enchaînement. Paris : Éditions du Seuil.

Eychenne, J. (2011). La liaison en français et la théorie de l'optimalité. Langue française, 169, $79-101$.

Eychenne, J., Lyche, C., Durand, J., et Coquillon, A. (2014). Quelles données pour la liaison aujourd'hui: la question des corpus. La liaison: approches contemporaines, éd par C. Soum-Favaro, A. Coquillon, et J.-P. Chevrot, 33-60. Berne: Peter Lang.

Lodge, A., Armstrong, N., Ellis, Y. M. L., et Shelton, J. F. (1997). Exploring the French Language. London : Arnold.

Malécot, A. (1975). French Liaison as a Function of Grammatical, Phonetic and Paralinguistic Variables. Phonetica, 32, 161-79.

Mallet, G.-M. (2008). La liaison en français : descriptions et analyses dans le corpus PFC. Thèse de doctorat, Université Paris Ouest Nanterre La Défense.

Meinschaefer, J., Bonifer, S., et Frisch, C. (2015). Variable and invariable liaison in a corpus of spoken French: Journal of French Language Studies, 25(3), 367-96.

Nardy, A. (2008). Acquisition des variables sociolinguistiques entre 2 et 6 ans: facteurs sociologiques et influences des interactions au sein du réseau social. Thèse doctorale, Université Stendhal - Grenoble III.

Pagliano, C. et Laks, B. (2005). Problématique de la liaison dans l'analyse d'un corpus de français oral actuel. Français fondamental, corpus oraux, contenus d'enseignement. 50 ans de travaux et d'enjeux, 8-10 décembre 2005, École Normale Supérieure Lettres et Science Humaines, Lyon, France. http://colloqueff.enslsh.fr/pdf/Laks_Bernard_Pagliano_Claudine.pdf.

Pope, M. K. (1934). From Latin to Modern French with Especial Consideration of AngloNorman: Phonology and Morphology. Manchester: Manchester University Press. 
Ranson, D. (2008). La liaison variable dans un corpus du français méridional : L'importance relative de la fonction grammaticale. Congrès Mondial de Linguistique Française, Recueil des résumés et CD-ROM des actes, éd par J. Durand, B. Habert, et B. Laks, 1657-71. Paris : Institut de Linguistique Française et EDP Sciences.

Segre, C., Tyssens, M., et Guidot, B. (2003). La Chanson de Roland, édition critique. Genève ; Droz.

Thurot, C. (1881). De la prononciation française depuis le commencement du XVI siècle, d'après les témoignages des grammairiens. Paris : Bibliothèque Nationale.

Valdman, A. (1993). Bien Entendu! Introduction à la prononciation française. Englewood Cliffs, NJ : Prentice Hall.

Walhout, N. (2017). Les facteurs linguistiques et sociolinguistiques en jeu dans la réalisation de la liaison en Afrique. Une comparaison entre la France hexagonale et l'Afrique. Mémoire de licence, Universiteit Utrecht.

Walter, H. (1988). Le français dans tous les sens. Paris : Robert Lafont.

${ }^{1}$ Cette locutrice appartient au sous-corpus Puteaux-Courbevoie et a été domiciliée en Île-deFrance au moment de l'enregistrement.

${ }^{2}$ Nous sommes conscientes du fait que le nombre inégal de liaisons entre les locuteurs du nord et du sud pourrait avoir un effet sur les résultats. Dans une future étude, nous analyserons toutes les liaisons dans dix minutes de conversation pour tous les locuteurs du nord.

${ }^{3}$ Les initiales indiquent les groupes de locuteurs comparés dans chaque colonne. Par exemple, $\mathrm{N}$ 0,870 dans la colonne « âge » est la valeur $p$ pour les locuteurs du nord selon l'âge, c'est-à-dire pour la comparaison entre le taux d'élision des jeunes et des personnes âgées du nord. NJ 0,906 dans la colonne "sexe » est une comparaison en fonction du sexe du locuteur pour les jeunes locuteurs du nord et ainsi de suite.

${ }^{4}$ Nous sommes d'accord avec notre relecteur qui a observé que l'amuïssement de la consonne [t] dans c'est-à-dire pourrait s'expliquer comme une réduction segmentale d'un mot fréquent et non pas comme la non-réalisation d'une liaison. De toute façon, nous avons trouvé intéressante cette comparaison entre les deux régions. L'inclusion de cette liaison n'ai sans doute pas un grand effet sur les résultats vu son faible nombre d'occurrences. 